\title{
Special Posters
}

\section{Back Pain (Lumbar Degenerative)}

\author{
Clinico-radiological outcomes following transforaminal versus lateral \\ lumbar interbody fusion \\ Fady Sedra, Arash Afsharpad, Benan Dala-Ali, Robert Lee, \\ Lester Wilson; Royal National Orthopaedic Hospital, Stanmore HA7 4LP, \\ $U K$
}

BACKGROUND CONTEXT: Lateral approach to lumbar fusion has been gaining popularity in recent years. It utilises a retroperitoneal transpsoas approach to reach the disc space in a minimally invasive fashion. It allows better access for disc clearance and insertion of large foot print cages.

PURPOSE: To compare radiographic and clinical results following lateral lumbar interbody fusion (LLIF) and minimally invasive transforaminal interbody fusion (MI-TLIF).

STUDY DESIGN/SETTING: Retrospective review.

PATIENT SAMPLE: We included 60 patients operated at 82 levels: Group 1 LLIF ( $\mathrm{n}=28$ ), Group 2 MI-TLIF $(\mathrm{n}=32)$. We measured radiological outcomes at $\mathrm{L} 3 / \mathrm{L} 4$ or $\mathrm{L} 4 / \mathrm{L} 5$.

OUTCOME MEASURES: Radiological: Disc height, foraminal height, segmental lordosis, overall lumbar lordosis. Clinical outcomes included visual analogue scores (VAS), Oswestry Disability Index (ODI) and European Quality of Life-5 Dimensions (EQ-5D).

METHODS: Radiological outcomes were measured on standing lateral X-rays preoperatively, immediately postoperatively and at the last follow up. Clinical outcomes were recorded preoperatively, at 6-8 weeks postoperatively and at the last follow-up.

RESULTS: Group 1: Mean improvement in disc height $9.1 \mathrm{~mm}$, foraminal height $5 \mathrm{~mm}$, segmental lordosis $6.75^{\circ}$, lumbar lordosis $15.4^{\circ}$, ODI 37 , VAS 56.2, EQ-5D 54. Group 2: Disc height $5.8 \mathrm{~mm}$, foraminal height $3.5 \mathrm{~mm}$, segmental lordosis $4.5^{\circ}$, overall lordosis 10.3, ODI 18, VAS 26, EQ-5D 23.5. Difference in parameters was statistically significant $(\mathrm{p}<.05)$ apart from ODI CONCLUSIONS: Cages inserted via the lateral approach have the ability to improve lumbar/segmental lordosis and is superior in restoring disc and foraminal height compared to MI-TLIF. Patients in the LLIF group showed improved clinical outcomes compared to the MI-TLIF group.

CONFLICTS OF INTEREST: No conflict of interest.

FUNDING SOURCES: No funding obtained.

http://dx.doi.org/10.1016/j.spinee.2016.01.150

Interbody fusion in low-grade spondylolisthesis-clinical outcome does not correlate with slip reduction and neural foraminal dimension Ujjwal K. Debnath, J.R. McConnell, A. Chatterjee,

Vivek Goel; Ramakrishna Mission Seva Pratisthan, 99 Sarat Bose Road, Kolkata 700026, India

BACKGROUND CONTEXT: Beneficial effect of reduction on outcome can be expected following reduction and fusion for low-grade lumbar spondylolisthesis but it is debatable.

PURPOSE: Analysis of correlation between the clinical outcome and radiological parameters.

STUDY DESIGN/SETTING: Prospective study.

PATIENT SAMPLE: Patients with low-grade spondylolisthesis who underwent transforaminal lumbar interbody fusion (TLIF).
OUTCOME MEASURES: Clinical outcome was measured by visual analogue scores (VAS) and Oswestry Disability Index (ODI); foraminal dimensions and disc heights were measured in standard digital radiographs. METHODS: 46 patients (age 17-48 years) with isthmic spondylolisthesis underwent interbody fusion with TLIF. Pre- and 1-year post-operative outcomes were analysed.

RESULTS: The baseline and at 1 year postoperative, the mean VAS scores were 6.39 (range 5-8) and 0.81 (range $0-3$ ) respectively ( $\mathrm{p}=.004$ ). The baseline and at 1 year postoperative, the mean ODI scores were 48 (range 3458 ) and 9.8 (range 4-22) respectively $(\mathrm{p}<.001)$. A mean spondylolisthesis slip of $33.2 \%$ was reduced to $6.7 \%$ at 1 year. Average anterior disc height, posterior disc height, $\mathrm{C}$ distance, $\mathrm{F}$ diameter improved from $9.4 \mathrm{~mm}$ to $11.7 \mathrm{~mm}(\mathrm{p}=.005)$, from $4.3 \mathrm{~mm}$ to $5.8 \mathrm{~mm}(\mathrm{p}=.004)$, from $11.2 \mathrm{~mm}$ to $12.0 \mathrm{~mm}(\mathrm{p}=.002)$, from $18.5 \mathrm{~mm}$ to $19.4 \mathrm{~mm}$ respectively $(\mathrm{p}<.001)$. Pearson correlation coefficients were significant for two radiological parameters, ie $(\Delta)$ listhesis $(r=0.46, p=.006)$ and $\mathrm{C}$ distance $(r=0.44, p=.01)$ for the improvement in the VAS scores only. All other radiological parameters had very low correlation with the clinical outcomes. Therefore, no correlation could be established statistically between the slip reduction and clinical outcome.

CONCLUSIONS: Neural decompression and interbody fusion significantly improved pain and disability, but the clinical outcome does not correlate with radiological improvement in neural foraminal dimension.

CONFLICTS OF INTEREST: None.

FUNDING SOURCES: None.

http://dx.doi.org/10.1016/j.spinee.2016.01.151

Feasibility of manufacturing a patient-specific spinal implant Tiziano Serra, Claudio Capelli, Deepak Kalaskar, Julian Leong; The Royal National Orthopaedic Hospital, University College London, London, UK

BACKGROUND CONTEXT: Spinal fusion is performed for degenerative spinal condition when conservative measures fail. Implant size and shape are not standardised between manufacturers, and best match often means compromises. Bioprinting offers a unique opportunity to create a tailormade solution.

PURPOSE: The goal of this study was to design and manufacture a 3Dprinted lumbar cage for lumbar interbody fusion.

STUDY DESIGN/SETTING: Feasibility study, laboratory based.

PATIENT SAMPLE: Nil.

OUTCOME MEASURES: Nil.

METHODS: Previous optimisation of the cage design via computational analysis was performed. Bioprinting process consists of a robotic tool enabling a layer-by-layer deposition of material to reproduce the designed cage. In order to print the cage, a low-cost 3D-printer was used. First, all the parameters of the process (eg temperature, deposition speed, filling density) were determined experimentally. Then, assessments of morphological, structural and mechanical properties were performed. Finally, biocompatibility was evaluated in vitro by assessing cell morphology, adhesion and proliferation. RESULTS: Computational analysis was a powerful method for preliminary testings of cage design and filling density. Surface evaluation showed a unique topographical pattern at the micro and nanoscale, and micro computed tomography (CT) scan confirmed structural reproducibility. Compressive tests showed comparison mechanical properties between trabecular bone and 
the printed cage. Finally, positive cell response proved high biocompatibility of the material used. Combination of optimised fabrication parameters gave a printing time of 2 hours and cost around $20 \mathrm{p}$ for each printed cage. CONCLUSIONS: Bioprinting is both feasible and affordable to offer patientspecific spinal implants.

CONFLICTS OF INTEREST: No conflicts of interest.

FUNDING SOURCES: Engineering and Physical Sciences Research Council (EPSRC) Centre for Innovative Manufacturing in Medical Devices (MeDe).

http://dx.doi.org/10.1016/j.spinee.2016.01.152

\section{Cervical Spine}

Berry ligament and the inferior thyroid artery as reliable anatomical landmarks for the recurrent laryngeal nerve (RLN)—a fresh cadaveric study relevant to the cervical spine Ali Rajabian, Michael Walsh, Nasir A. Quraishi; The Centre for Spinal Studies and Surgery, Queen's Medical Centre, Nottingham NG7 2UH, UK

BACKGROUND CONTEXT: Whilst most cadaveric studies of the recurrent laryngeal nerve (RLN) have focused on course variations as a suitable guide for right versus left RLN, they have mostly been done on preserved (fixed) cadavers that render the RLN immobile.

PURPOSE: Our aim was to perform anterior cervical exposures from $\mathrm{C} 2$ to T2/T3 with particular attention to the course of the RLN on right- and left-sided exposures in fresh cadaveric specimens. In addition, we aimed to expose the entire course of the RLN. Finally, we wanted to show the position of the RLN in relation to the trachea-oesophageal groove, inferior thyroid artery and Berry ligament.

STUDY DESIGN/SETTING: Fresh cadaveric study.

PATIENT SAMPLE: Eight fresh cadavers.

OUTCOME MEASURES: High-quality photographs of cadaveric study. METHODS: Eight fresh cadavers had extensive layer-by-layer dissections performed by two surgeons (one of whom has extensive experience as an anatomy demonstrator and dissector). The RLNs were exposed in their entire length and relationship to different landmarks recorded. Photographs were taken at each stage of the exposure.

RESULTS: In all specimens, we were able to demonstrate the entire course of both RLNs from origin to insertion. The RLNs were consistently associated with the inferior thyroid artery and Berry ligament bilaterally with the RLNs passing at almost perpendicular to these structures.

CONCLUSIONS: The near horizontal direction of Berry ligament in the cervical tissue planes exposed during anterior cervical exposures enables the surgeon to reliably identify the expected position of RLN at its medial end and hence avoid it prior to visual observation of the nerve on either side. We found that the most reliable anatomical landmark bilaterally for the RLN was the inferior thyroid artery and Berry ligament, both of which would be encountered in anterior surgical exposure prior to the nerve itself. We believe that this will help spinal surgeons refine their surgical technique to identify this nerve where necessary and thus reduce the incidence of iatrogenic injury. CONFLICTS OF INTEREST: No conflicts of interest.

FUNDING SOURCES: No funding obtained.

http://dx.doi.org/10.1016/j.spinee.2016.01.153

Right- versus left-sided exposures of the recurrent laryngeal nerve (RLN) and its branches-a fresh cadaveric study relevant to the cervical spine

Ali Rajabian, Michael Walsh, Nasir A. Quraishi; The Centre for Spinal Studies and Surgery, Queen's Medical Centre, Nottingham NG7 2UH, UK

BACKGROUND CONTEXT: The higher vulnerability of RLN in anterior approach to the cervical spine on the right versus left sides is the subject of ongoing debate. Whilst most cadaveric studies have focused on in situ variations in course and local structural relations of the RLN as a suitable guide, they have mostly been done in preserved (fixed) cadavers or without relevance to the needs of spinal exposure.

PURPOSE: Our aim was to perform surgically relevant exposures of the anterior cervical spine, with particular attention to observing the potential vulnerabilities of the RLN on the right and left sides in fresh cadaveric specimens. In addition, we aimed to explore its branches.

STUDY DESIGN/SETTING: Fresh cadaveric study.

PATIENT SAMPLE: Eight fresh cadavers.

OUTCOME MEASURES: High-quality photographs of cadaveric study. METHODS: Twelve cadavers had extensive layer-by-layer dissections by two surgeons (one with extensive experience as anatomy dissector). The RLNs and their branches were exposed in their entire length and explored for vulnerability. (Each stage was photographed.)

RESULTS: In all specimens, we demonstrated that right-sided approach clearly causes undue stretch of the nerve and its branches particularly below C5 (photographed). The left side provided a good exposure without undue traction on the nerve. However, the terminal oesophageal branches of the nerve were especially vulnerable to this stretch or direct pressure on the left side.

CONCLUSIONS: Traction neuropraxia of the upper oesophageal branches of the RLN may provide an alternative explanation for the transient postoperative dysphagia (up to 60\%). The laryngeal supply of the RLN benefits from Galen anastomotic nerve supply of superior laryngeal nerve (SLN) (Ansa of Galen) and hence the neuropraxia of the nerve is less frequently symptomatic. Below C5, the left-sided anterior cervical approach permits a wider access to the entire anterior cervical spine with less risk to the RLN. Neuropraxia induced on the oesophageal branches (directly by retractors or indirectly by traction) could provide a possible explanation for transient post-operative dysphagia. We believe that this will help spinal surgeons to refine their surgical technique and thus reduce the incidence of iatrogenic injury.

CONFLICTS OF INTEREST: No conflicts of interest.

FUNDING SOURCES: No funding obtained.

http://dx.doi.org/10.1016/j.spinee.2016.01.154

Comparing patient outcome measures in a cohort of patients who underwent anterior cervical discectomy and fusion (ACDF) versus cage-plate ACDF

Shrijit Panikkar, Iain McLaughlin-Symon, Gagan Sethi, Irfan Siddique; Spinal Unit, Salford Royal NHS Foundation Trust, Stott Lane, Manchester M6 8HD, UK

BACKGROUND CONTEXT: Most studies tend to favour better fusion rates with anterior cervical discectomy and fusion with plating against without. It is unclear whether this translates to better patient outcome.

PURPOSE: Comparing patient outcomes of stand-alone cage anterior cervical discectomy and fusion (ACDF) versus cage-plate ACDF.

STUDY DESIGN/SETTING: Retrospective study of prospectively collected registry data.

PATIENT SAMPLE: Consecutive patients undergoing ACDF over a 4-year period at a single centre.

OUTCOME MEASURES: Registry collected surgical details, global effectiveness score and Core Outcome Multidimensional Index (COMI) scores pre-operatively and at 1 year post-operatively.

METHODS: Statistical analysis of the outcome measures of the two groups was undertaken using the t-test and the chi-square test.

RESULTS: After exclusion of missing data pre-operative, surgical and 1 year post-operative data were available for 212 patients who underwent stand-alone cage ACDF and 34 patients who underwent cage-plate ACDF. A similar percentage [24 (71.59\%) and $152(71.03 \%)$ ] of patients in each group were satisfied with the outcome of the surgery. Both groups had similar global outcome, improvement in COMI scores and perioperative complication rates. 